\title{
Search for Axionlike Dark Matter through Nuclear Spin Precession in Electric and Magnetic Fields
}

C. Abel,${ }^{1}$ N. J. Ayres,,${ }^{1,}$ G. Ban, ${ }^{2}$ G. Bison,${ }^{3}$ K. Bodek,${ }^{4}$ V. Bondar,${ }^{5}$ M. Daum,${ }^{3}$ M. Fairbairn, ${ }^{6}$ V. V. Flambaum, ${ }^{7}$ P. Geltenbort, ${ }^{8}$ K. Green, ${ }^{9}$ W. C. Griffith, ${ }^{1}$ M. van der Grinten, ${ }^{9}$ Z. D. Grujić, ${ }^{10}$ P. G. Harris, ${ }^{1}$ N. Hild, ${ }^{3}$ P. Iaydjiev, ${ }^{9,}$ S. N. Ivanov, ${ }^{9,8}$ M. Kasprzak,${ }^{5}$ Y. Kermaidic, ${ }^{11}$ K. Kirch,${ }^{12,3}$ H.-C. Koch, ${ }^{3}$ S. Komposch, ${ }^{3,12}$ P. A. Koss, ${ }^{5}$ A. Kozela, ${ }^{13}$ J. Krempel, ${ }^{12}$ B. Lauss, ${ }^{3}$ T. Lefort, ${ }^{2}$ Y. Lemière, ${ }^{2}$ D. J. E. Marsh, ${ }^{6}$ P. Mohanmurthy, ${ }^{3,12}$ A. Mtchedlishvilii, ${ }^{3}$ M. Musgrave, ${ }^{1,9}$ F. M. Piegsa, ${ }^{14}$ G. Pignol, ${ }^{11}$ M. Rawlik, ${ }^{12, \dagger}$ D. Rebreyend, ${ }^{11}$ D. Ries,${ }^{14,3,12}$ S. Roccia, ${ }^{15}$ D. Rozpędzik, ${ }^{4}$ P. SchmidtWellenburg, ${ }^{3}$ N. Severijns, ${ }^{5}$ D. Shiers, ${ }^{1}$ Y. V. Stadnik, ${ }^{7}$ A. Weis, ${ }^{10}$ E. Wursten, ${ }^{5}$ J. Zejma, ${ }^{4}$ and G. Zsigmond ${ }^{3}$

${ }^{1}$ Department of Physics and Astronomy, University of Sussex, Falmer,

Brighton BN1 9QH, United Kingdom

${ }^{2}$ Normandie Univ, ENSICAEN, UNICAEN, CNRS/IN2P3, LPC Caen, 14000 Caen, France

${ }^{3}$ Paul Scherrer Institute, CH-5232 Villigen PSI, Switzerland

${ }^{4}$ Institute of Physics, Jagiellonian University in Kraków, 30-348 Kraków, Poland

${ }^{5}$ Instituut voor Kern- en Stralingsfysica, Katholieke Universiteit Leuven, B-3001 Leuven, Belgium

${ }^{6}$ King's College London Department of Physics, London, WC2R 2LS, United Kingdom

${ }^{7}$ School of Physics, University of New South Wales, Sydney 2052, Australia and Johannes Gutenberg University, 55122 Mainz, Germany

${ }^{8}$ Institut Laue-Langevin, F-38042 Grenoble Cedex 9, France

${ }^{9}$ Rutherford Appleton Laboratory, Chilton, Didcot, Oxon OX11 OQX, United Kingdom

${ }^{10}$ Physics Department, University of Fribourg, CH-1700 Fribourg, Switzerland

${ }^{11}$ LPSC, Université Grenoble Alpes, CNRS/IN2P3, 38026 Grenoble, France

${ }^{12}$ ETH Zürich, Institute for Particle Physics and Astrophysics, CH-8093 Zürich, Switzerland

${ }^{13}$ Institute of Nuclear Physics, Polish Academy of Sciences, 31-342 Kraków, Poland

${ }^{14}$ Laboratory for High Energy Physics, Albert Einstein Center for Fundamental Physics, University of Bern, $\mathrm{CH}-3012$ Bern, Switzerland

${ }^{15}$ CSNSM, Université Paris Sud, CNRS/IN2P3, Université Paris Saclay, Bat. 104\&108, F91405 Orsay-Campus, France

(Received 29 August 2017; published 14 November 2017)

\begin{abstract}
We report on a search for ultralow-mass axionlike dark matter by analyzing the ratio of the spinprecession frequencies of stored ultracold neutrons and ${ }^{199} \mathrm{Hg}$ atoms for an axion-induced oscillating electric dipole moment of the neutron and an axion-wind spin-precession effect. No signal consistent with dark matter is observed for the axion mass range $10^{-24} \leq m_{a} \leq 10^{-17} \mathrm{eV}$. Our null result sets the first laboratory constraints on the coupling of axion dark matter to gluons, which improve on astrophysical limits by up to 3 orders of magnitude, and also improves on previous laboratory constraints on the axion coupling to nucleons by up to a factor of 40 .
\end{abstract}

DOI: 10.1103/PhysRevX.7.041034

Subject Areas: Cosmology, Particles and Fields

\section{INTRODUCTION}

Astrophysical and cosmological observations indicate that $26 \%$ of the total energy density and $84 \%$ of the total matter content of the Universe is dark matter
(DM) [1], the identity and properties of which still remain a mystery. One of the leading candidates for cold DM is the axion, a pseudoscalar particle that was originally hypothesized to resolve the strong $C P$ problem of quantum

\footnotetext{
*Corresponding author.

N.Ayres@sussex.ac.uk

Corresponding author.

mrawlik@phys.ethz.ch

${ }^{*}$ Present address: Institute of Nuclear Research and Nuclear Energy, Sofia, Bulgaria.

${ }^{\S}$ Present address: Petersburg Nuclear Physics Institute, Russia.

"Present address: Laboratory for Nuclear Science, Massachusetts Institute of Technology, Cambridge, MA 02139, USA.
}

Published by the American Physical Society under the terms of the Creative Commons Attribution 4.0 International license. Further distribution of this work must maintain attribution to the author(s) and the published article's title, journal citation, and DOI. 
chromodynamics (QCD) [2-9]. Apart from the canonical QCD axion, various axionlike particles have also been proposed, for example, in string compactification models [10-15].

Low-mass $\left(m_{a} \lesssim 0.1 \mathrm{eV} / c^{2}\right)$ axions can be produced efficiently via nonthermal production mechanisms, such as vacuum misalignment [16-18] in the early Universe, and subsequently form a coherently oscillating classical field [19]: $a=a_{0} \cos (\omega t)$, with the angular frequency of oscillation given by $\omega \approx m_{a} c^{2} / \hbar$, where $m_{a}$ is the axion mass (henceforth, we adopt the units $\hbar=c=1$ ). The oscillating axion field carries the energy density $\rho_{a} \approx m_{a}^{2} a_{0}^{2} / 2$. Because of its effects on structure formation [20], ultralow-mass axion DM in the mass range $10^{-24} \lesssim m_{a} \lesssim$ $10^{-20} \mathrm{eV}$ has been proposed as a DM candidate that is observationally distinct from, and possibly favorable to, archetypal cold DM [15,21-24]. The requirement that the axion de Broglie wavelength does not exceed the DM size of the smallest dwarf galaxies and consistency with observed structure formation [25-27] give the lower axion mass bound $m_{a} \gtrsim 10^{-22} \mathrm{eV}$, if axions comprise all of the DM. However, axions with smaller masses can constitute a subdominant fraction of DM [28].

It is reasonable to expect that axions interact nongravitationally with standard-model particles. Direct searches for axions have thus far focused mainly on their coupling to the photon (see the review in Ref. [29] and references therein). Recently, however, it has been proposed to search for the interactions of the coherently oscillating axion DM field with gluons and fermions, which can induce oscillating electric dipole moments (EDMs) of nucleons [30] and atoms [31-33], and anomalous spin-precession effects $[31,34,35]$. The frequency of these oscillating effects is dictated by the axion mass, and more importantly, these effects scale linearly in a small interaction constant [30-35], whereas in previous axion searches, the sought effects scaled quadratically or quartically in the interaction constant [29].

In the present work, we focus on the axion-gluon and axion-nucleon couplings:

$$
\mathcal{L}_{\text {int }}=\frac{C_{G}}{f_{a}} \frac{g^{2}}{32 \pi^{2}} a G_{\mu \nu}^{b} \tilde{G}^{b \mu \nu}-\frac{C_{N}}{2 f_{a}} \partial_{\mu} a \bar{N} \gamma^{\mu} \gamma^{5} N,
$$

where $G$ and $\tilde{G}$ are the gluonic field tensor and its dual, $b=1,2, \ldots, 8$ is the color index, $g^{2} / 4 \pi$ is the color coupling constant, $N$ and $\bar{N}=N^{\dagger} \gamma^{0}$ are the nucleon field and its Dirac adjoint, $f_{a}$ is the axion decay constant, and $C_{G}$ and $C_{N}$ are model-dependent dimensionless parameters. Astrophysical constraints on the axion-gluon coupling in Eq. (1) come from big bang nucleosynthesis [36-38], $m_{a}^{1 / 4} f_{a} / C_{G} \gtrsim$ $10^{10} \mathrm{GeV}^{5 / 4}$ for $m_{a} \ll 10^{-16} \mathrm{eV}$ and $m_{a} f_{a} / C_{G} \gtrsim$ $10^{-9} \mathrm{GeV}^{2}$ for $m_{a} \gg 10^{-16} \mathrm{eV}$, assuming that axions saturate the present-day DM energy density, and from supernova energy-loss bounds [35,39], $f_{a} / C_{G} \gtrsim 10^{6} \mathrm{GeV}$ for $m_{a} \lesssim 3 \times 10^{7} \mathrm{eV}$. Astrophysical constraints on the axion-nucleon coupling in Eq. (1) come from supernova energy-loss bounds [39,40], $f_{a} / C_{N} \gtrsim 10^{9} \mathrm{GeV}$ for $m_{a} \lesssim 3 \times 10^{7} \mathrm{eV}$, while existing laboratory constraints come from magnetometry searches for new spin-dependent forces mediated by axion exchange [41], $f_{a} / C_{N} \gtrsim$ $1 \times 10^{4} \mathrm{GeV}$ for $m_{a} \lesssim 10^{-7} \mathrm{eV}$.

The axion-gluon coupling in Eq. (1) induces the following oscillating EDM of the neutron via a chirally enhanced one-loop process [42-44]:

$$
d_{n}(t) \approx+2.4 \times 10^{-16} \frac{C_{G} a_{0}}{f_{a}} \cos \left(m_{a} t\right) e \mathrm{~cm} .
$$

The axion-gluon coupling in Eq. (1) also induces oscillating EDMs of atoms via the one-loop-level oscillating nucleon EDMs and tree-level oscillating $P-, T$-violating intranuclear forces (which give the dominant contribution) $[31,45]$. In the case of ${ }^{199} \mathrm{Hg}$, the oscillating atomic EDM is $[31,37,46-53]$

$$
d_{\mathrm{Hg}}(t) \approx+1.3 \times 10^{-19} \frac{C_{G} a_{0}}{f_{a}} \cos \left(m_{a} t\right) e \mathrm{~cm},
$$

which is suppressed compared to the value for a free neutron [Eq. (2)], as a consequence of the Schiff screening theorem for neutral atoms [54]. The amplitude of the axion DM field $a_{0}$ is fixed by the relation $\rho_{a} \approx m_{a}^{2} a_{0}^{2} / 2$. In the present work, we assume that axions saturate the local cold DM energy density $\rho_{\mathrm{DM}}^{\text {local }} \approx 0.4 \mathrm{GeV} / \mathrm{cm}^{3}$ [55].

The derivative coupling of an oscillating galactic axion DM field, $a=a_{0} \cos \left(m_{a} t-\boldsymbol{p}_{a} \cdot \boldsymbol{r}\right)$, with spin-polarized nucleons in Eq. (1) induces time-dependent energy shifts according to

$$
H_{\mathrm{int}}(t)=\frac{C_{N} a_{0}}{2 f_{a}} \sin \left(m_{a} t\right) \boldsymbol{\sigma}_{N} \cdot \boldsymbol{p}_{a} .
$$

The term $\boldsymbol{\sigma}_{N} \cdot \boldsymbol{p}_{a}$ is conveniently expressed by transforming to a nonrotating celestial coordinate system (see, e.g., Ref. [56]):

$$
\begin{aligned}
\boldsymbol{\sigma}_{N} \cdot \boldsymbol{p}_{a}= & \hat{m}_{F} f\left(\sigma_{N}\right) m_{a}\left|\boldsymbol{v}_{a}\right| \\
& \times\left[\cos (\chi) \sin (\delta)+\sin (\chi) \cos (\delta) \cos \left(\Omega_{\text {sid }} t-\eta\right)\right],
\end{aligned}
$$

where $\chi$ is the angle between Earth's axis of rotation and the spin quantization axis $\left[\chi=42.5^{\circ}\right.$ at the location of the Paul Scherrer Institute (PSI)], $\delta \approx-48^{\circ}$ and $\eta \approx 138^{\circ}$ are the declination and right ascension of the galactic axion DM flux relative to the Solar System [57], $\Omega_{\text {sid }} \approx$ $7.29 \times 10^{-5} \mathrm{~s}^{-1}$ is the daily sidereal angular frequency, $\hat{m}_{F}=m_{F} / F$ is the normalized projection of the total 
angular momentum onto the quantization axis, and $f\left(\sigma_{N}\right)=+1$ is the nucleon spin content function for the free neutron, while $f\left(\sigma_{N}\right)=-1 / 3$ for the ${ }^{199} \mathrm{Hg}$ atom in the Schmidt (single-particle) model [58].

Here, we report on a search for an axion-induced oscillating EDM of the neutron (nEDM) based on an analysis of the ratio of the spin-precession frequencies of stored ultracold neutrons and ${ }^{199} \mathrm{Hg}$ atoms, which is a system that had previously also been used as a sensitive probe of new non-EDM physics [59-61]. We divide our analysis into two parts. We first analyze the Sussex-RALILL nEDM experiment data [62], covering oscillation periods longer than days (long-time base). Then we extend the analysis to the data of the PSI nEDM experiment [63], which allows us to probe oscillation periods down to minutes (short-time base). Our analysis places the first laboratory constraints on the axion-gluon coupling. We also report on a search for an axion-wind spin-precession effect, using the data of the PSI nEDM experiment. Our analysis places the first laboratory constraints on the axion-nucleon coupling from the consideration of an effect that is linear in the interaction constant.

\section{LONG-TIME-BASE ANALYSIS}

The Sussex-RAL-ILL room-temperature nEDM experiment ran from 1998 to 2002 at the PF2 beam line at the Institut Laue-Langevin (ILL) in Grenoble, France. This experiment set the current world-best limit on the permanent time-independent neutron EDM, published in 2006 [64]. The data were subsequently reanalyzed to give a revised limit in 2015 [65]. The technical details of the apparatus are described in full in Ref. [62], but we summarize the main experimental details here for the reader.

The experiment was based on Ramsey interferometry [66] of ultracold neutrons [67,68]. The neutrons were stored in parallel or antiparallel electric and magnetic fields, where their Larmor precession frequency is given by

$$
h \nu_{n}=2\left|\mu_{n} B \pm d_{n} E\right|,
$$

with the sign depending on the field configuration. $E$ and $B$ are the magnitudes of the electric and magnetic fields, respectively. By measuring the frequency difference between the two field configurations, a value for the neutron $\mathrm{EDM} d_{n}$ was inferred.

The measurement was conducted in a series of cycles, each approximately 5 min long. A cycle began with a filling of neutrons polarized along the fields into the precession chamber from the ultracold neutron source [69]. Once they are in the chamber, enclosed from top and bottom with electrodes, a $29-\mathrm{Hz}$ NMR pulse lasting $2 \mathrm{~s}$ was applied to rotate the neutron spins into the transverse plane of the electromagnetic fields where they began to precess. Prior to the pulse, a population of polarized ${ }^{199} \mathrm{Hg}$ atoms was released into the chamber, and an $8-\mathrm{Hz}$ NMR pulse was applied to set these atoms into free precession. The particles were stored and allowed to precess freely for $130 \mathrm{~s}$. After this, another 2-s $29-\mathrm{Hz}$ pulse, in phase with the first one, was applied.

The neutrons were then emptied into a detector through a spin-analyzing foil. Over 1-2 days, many of these cycles were performed. The electric field's polarization was reversed every hour. We term one continuous block of data taking in the same magnetic-field configuration, but including both directions of electric field, a run. One run gives a $d_{n}$ estimate.

In order to suppress cycle-to-cycle changes in the magnetic field, the analysis was performed on the ratio of the neutron and mercury precession frequencies $R$, which, using Eq. (6), is [62]

$$
R \equiv \frac{\nu_{n}}{\nu_{\mathrm{Hg}}}=\frac{\mu_{n}}{\mu_{\mathrm{Hg}}} \pm\left(d_{n}-\frac{\mu_{n}}{\mu_{\mathrm{Hg}}} d_{\mathrm{Hg}}\right) \frac{2 E}{h \nu_{\mathrm{Hg}}}+\Delta,
$$

where the signs correspond to parallel and antiparallel field configurations. $\Delta$ encapsulates all higher-order terms and systematic effects, which are corrected for when a run is analyzed [65]. This analysis is sensitive to oscillations in the quantity $d_{n}-\left(\mu_{n} / \mu_{\mathrm{Hg}}\right) d_{\mathrm{Hg}}$, with $\mu_{n} / \mu_{\mathrm{Hg}}=$ $-3.8424574(30)$ [70].

In our analysis, we are looking for an oscillating EDM. We perform this search in frequency space by evaluating periodograms-estimators of the power spectrum. An oscillation in the time domain shows up as an excess in the power (or, equivalently, amplitude) relative to the expected distribution due to experimental noise.

In the case of the long-time-base analysis, we consider the time series of $d_{n}$ measurements from individual runs (after having corrected for the "false EDM" effect [71] using the crossing lines procedure [65]). The measurements are neither evenly spaced nor have equal uncertainties. To calculate the periodogram of the data series, we use the least squares spectral analysis (LSSA) method [72,73], where the amplitude at frequency $f$ is estimated by the amplitude of the best-fit oscillation of that frequency. We evaluate the periodogram at a set of 1334 trial frequencies, evenly spaced between $100 \mathrm{pHz}$ (arbitrarily chosen, a period of about 300 years, much longer than the 4-year span of the data set) and $10 \mu \mathrm{Hz}$ (a period of about a day, the time it typically took to get one $d_{n}$ estimate). An axion DM signal, with expected coherence set by $\Delta f \sim 10^{-6} f$ [19], is narrower than the spectral resolution $(7.49 \mathrm{nHz}$, the inverse span of the data set) in the whole range of frequencies we are sensitive to. In the LSSA fit, we assume the free offset to be zero on the grounds that the experiment has already delivered a zero-compatible result for the permanent time-independent neutron EDM $[64,65]$. The periodogram of the long-time-base data set is shown as a black line in Fig. 1. To obtain the expected distribution of 


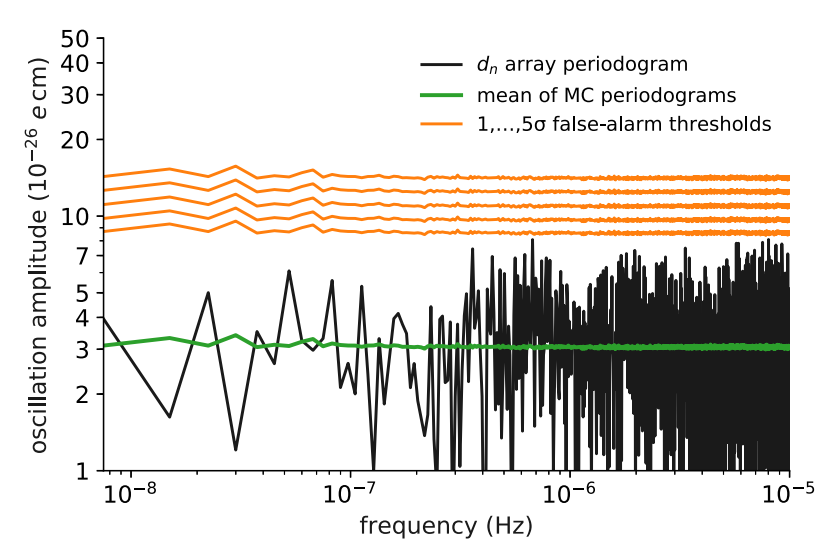

FIG. 1. The periodogram of the array of neutron EDM $\left(d_{n}\right)$ estimates from the ILL measurement (black line). We are sensitive to oscillations in the quantity $d_{n}-\left(\mu_{n} / \mu_{\mathrm{Hg}}\right) d_{\mathrm{Hg}}$, where $d_{\mathrm{Hg}}$ is the EDM of the ${ }^{199} \mathrm{Hg}$ atom. The mean of Monte Carlogenerated periodograms, assuming no signal is present, is depicted in green. MC simulation is used to deliver false-alarm thresholds (global $p$ values), marked in orange for $1,2, \ldots, 5 \sigma$ levels (from bottom to top). The highest peak has the global $p$ value 0.53 , consistent with a nondetection.

the periodogram, we perform Monte Carlo (MC) simulations. At each frequency, we estimate the cumulative distribution function (CDF) of the LSSA power. Extreme events in the tails of the distribution are expensive to access directly with MC methods. For this reason, to the discrete CDF estimates we fit, at each $i$ th frequency, the functional form of the LSSA-power CDF [72]:

$$
F_{i}(\mathcal{P})=1-A_{i} \exp \left(-B_{i} \mathcal{P}\right)
$$

where $\mathcal{P}$ is the power, while $A_{i}$ and $B_{i}$ are fit parameters. The local $p$ values are given by

$$
p_{\text {local }, i}=1-F_{i}\left(\mathcal{P}_{i}\right),
$$

where $\mathcal{P}_{i}$ is the LSSA power of the measured $d_{n}$ time series at the $i$ th frequency.

If the local $p$ values at different trial frequencies were uncorrelated, the global $p$ value would be given by [74]

$$
p_{\text {global }}=1-\left(1-p_{\text {local }}\right)^{N},
$$

where $N$ is the number of trial frequencies. However, we do not need to make this assumption. Instead, we make use of the set of MC data sets. In each, we find the minimal local $p$ value and estimate its $\mathrm{CDF}$, assuming it has the form Eq. (10), but leave $N$ as a free parameter. We find the bestfit value, $N_{\text {effective }}=1026$. For each frequency, we mark the power necessary to reach the global $p$ values corresponding to $1,2, \ldots, 5 \sigma$ levels as orange lines in Fig. 1 . The minimal local $p$ value of the data set translates to the global $p$ value of 0.53 , consistent with a nondetection.

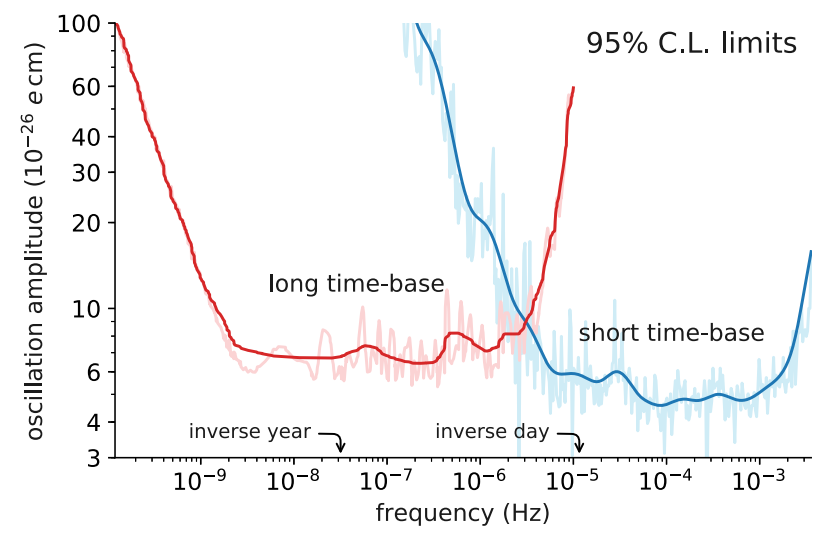

FIG. 2. The 95\% C.L. limits on the amplitude of oscillation in the quantity $d_{n}-\left(\mu_{n} / \mu_{\mathrm{Hg}}\right) d_{\mathrm{Hg}}$, as a function of frequency thereof. The limits from the long-time-base (ILL data) and short-time-base (PSI data) analyses are depicted by the red and blue curves, respectively, with the area above these curves being excluded. The raw limits delivered by the analysis, with substantial noise, are depicted by the light lines, while the smoothed versions are given in bold.

In order to obtain limits on the oscillation amplitude parameter, we again use MC simulations. We discretize the space of possible signals, spanned by their frequency and amplitude. We choose a sparser set of 200 frequencies, as we do not expect highly coherent effects in the sensitivity of detection. For each discrete point, we generate a set of 200 MC data sets containing the respective, perfectly coherent signal and assume that the oscillation is averaged over the duration of the run. In general, the sensitivity is phase dependent, especially for periods comparable with the length of the data set. For simplicity, we do not investigate the phase dependence and in the simulation take it to be random and uniformly distributed. For each fake data set, we evaluate the LSSA amplitude only at the frequency of the signal and compare its distribution [extrapolating with the functional form of Eq. (8)] with the best-fit amplitude in the data and define the $p$ value to be left-sided. We find the $95 \%$ confidence-level exclusion limit as the 0.05 isocontour of the $\mathrm{CL}_{s}$ statistic [75]. The limit is shown as the red curve in Fig. 2. We are most sensitive to periods shorter than the time span of the data set ( $\sim 4$ years), but rapidly lose sensitivity for periods shorter than the temporal spacing between data points ( $\sim 2$ days), since the expected signal would essentially average to zero over these short time scales.

\section{SHORT-TIME-BASE ANALYSIS}

In 2009, the Sussex-RAL-ILL apparatus was moved to the new ultracold neutron source at the Paul Scherrer Institute, Villigen, Switzerland [76-79], where a number of improvements were made $[63,80,81]$. In 2015, the apparatus was fully commissioned and began to take high-sensitivity EDM data. The whole data set, taken from 
August 2015 until the end of 2016, with a higher accumulated sensitivity than the ILL one, was considered in this analysis. For the PSI experiment's data, we perform a lower-level oscillation search on the array of $R$ measurements. Since an $R$ estimate was obtained every cycle $(\approx 300 \mathrm{~s}, 180 \mathrm{~s}$ of which was the free precession), rather than every 1-2 days as for a $d_{n}$ estimate, it has an increased sensitivity to higher frequencies. Additionally, the analysis could benefit from the addition of 16 atomic cesium vapor magnetometers $[82,83]$, located directly above and below the precession chamber (inside the electrodes). This made it possible to account for the dominant time-dependent systematic effect on a cycle, rather than run, basis.

The dominant time-dependent systematic effect, encapsulated in $\Delta$ of Eq. (7), would have given rise to nonstatistical temporal fluctuations if not accounted for. Namely, $R$ is sensitive to drifts in the vertical gradients of the magnetic field. While the thermal mercury atoms fills the chamber homogeneously, the center of mass of the ultracold neutron population is lower by several millimeters $[65,70,84]$. To evaluate the correction, the drifts of the gradients are estimated on a cycle basis by fitting a second-order parametrization of the magnetic field to the measurements of the cesium magnetometers [85]. The center-of-mass shift was determined to be $4 \mathrm{~mm}$ using the method described in Ref. [70].

The measurement procedure involves working deliberately with gradients affecting $R$ (see the crossing-point method in Ref. [65]). The intended gradients (up to $60 \mathrm{pT} / \mathrm{cm}$ in steps) are much larger than cycle-tocycle fluctuations $(<2 \mathrm{pT} / \mathrm{cm}$ per day). With the highorder shifts in $R$ being significant, these large shifts could not be corrected using the cesium magnetometers. Additionally, while the cesium magnetometers are precise, their accuracy is limited by the calibration procedure. We define as a sequence a set of data, typically $2-3$ days in duration, without a deliberate change in the magnetic-field gradient or a recalibration of the cesium magnetometers. When performing the LSSA fit, we allow the free offset to be different in each sequence:

$$
A \sin (2 \pi f t)+B \cos (2 \pi f t)+\sum_{i} C_{i} \Pi_{i}(t),
$$

where $C_{i}$ is the free offset in the $i$ th sequence and $\Pi_{i}(t)$ is a gate function equal to one in the $i$ th sequence and zero elsewhere. This causes the short-time-base analysis to lose sensitivity for periods longer than one sequence. It should also be mentioned that, at the time of this analysis, the PSI data were still blinded, whereby an unknown, but constant, $d_{n}$ was injected into them. It does not influence this analysis, as the free offsets are not considered further.

We split the $R$ time array into three sets: a control set of data without an applied electric field and two sets sensitive to an oscillating EDM, namely, with parallel and antiparallel applied electric and magnetic fields. A coherent oscillating
EDM signal would have an opposite phase in the latter two sets, and be absent in the control set. We do not perform a common fit. Instead, the two sensitive data sets are treated separately in the LSSA fits, and later combined to a limit. Otherwise, the LSSA treatment is the same as in the longtime-base analysis. We pick a set of 156198 trial frequencies, spaced apart at intervals determined by the spectral resolution (the inverse of 506 days $=23 \mathrm{nHz}$ ), which here also defines the signal width.

The periodogram of the $R$ time array taken with the parallel-field configuration is shown in black in Fig. 3. There are two regions of expected rise in the oscillation amplitude due to the time structure of the data collection. The one around $28 \mu \mathrm{Hz}$ (the inverse of $10 \mathrm{~h}$ ) corresponds to the period of the electric-field reversal. The very narrow one around $3.3 \mathrm{mHz}$ (the inverse of $300 \mathrm{~s}$ ) corresponds to the cycle repetition rate. There are five trial frequencies for which the $3 \sigma$ false-alarm threshold is exceeded, two of which, including the largest excess with a $6 \sigma$ significance, occur in a $100-\mu \mathrm{Hz}$ region around the inverse of $300 \mathrm{~s}$, while the other three are in the low-frequency region (inverse days) already excluded by the long-time-base analysis. The periodograms for the other two data sets (not shown) are very similar. In the other sensitive set, there are three excesses of the $3 \sigma$ threshold (the highest is $5 \sigma$ ), all constrained to the same two regions. In the control data set, only the $1 \sigma$ threshold is exceeded. The periodogram of the $R$ time array without the gradient-drift correction is shown in pink in Fig. 3 to visualize the frequencies where the correction has an effect.

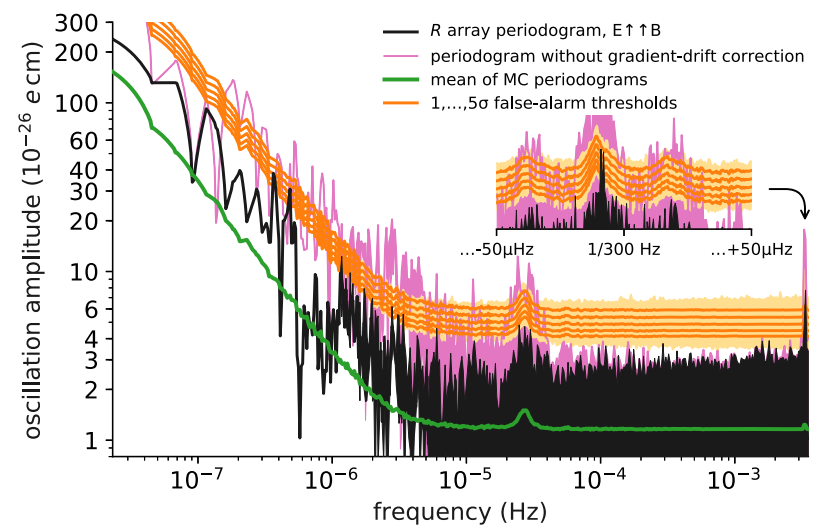

FIG. 3. Periodogram of the $R$ time array of the PSI experiment data, sensitive to oscillations in the quantity $d_{n}-\left(\mu_{n} / \mu_{\mathrm{Hg}}\right) d_{\mathrm{Hg}}$, taken with the $\boldsymbol{E}$ and $\boldsymbol{B}$ fields parallel (black line). The mean of $\mathrm{MC}$-generated periodograms, assuming no signal, is depicted in green. MC simulation is used to calculate $1,2, \ldots, 5 \sigma$ false-alarm thresholds, depicted in light orange. For clarity, we also plot the smoothed version in orange. There are two regions where a rise in the amplitude is expected, namely, around $28 \mu \mathrm{Hz}$ (inverse of $10 \mathrm{~h}$ ) and $3.3 \mathrm{mHz}$ (inverse of $300 \mathrm{~s}$ ), due to the time structure of the data taking (see the main text for more details). The periodogram of nongradient-drift-corrected data is shown in pink. 

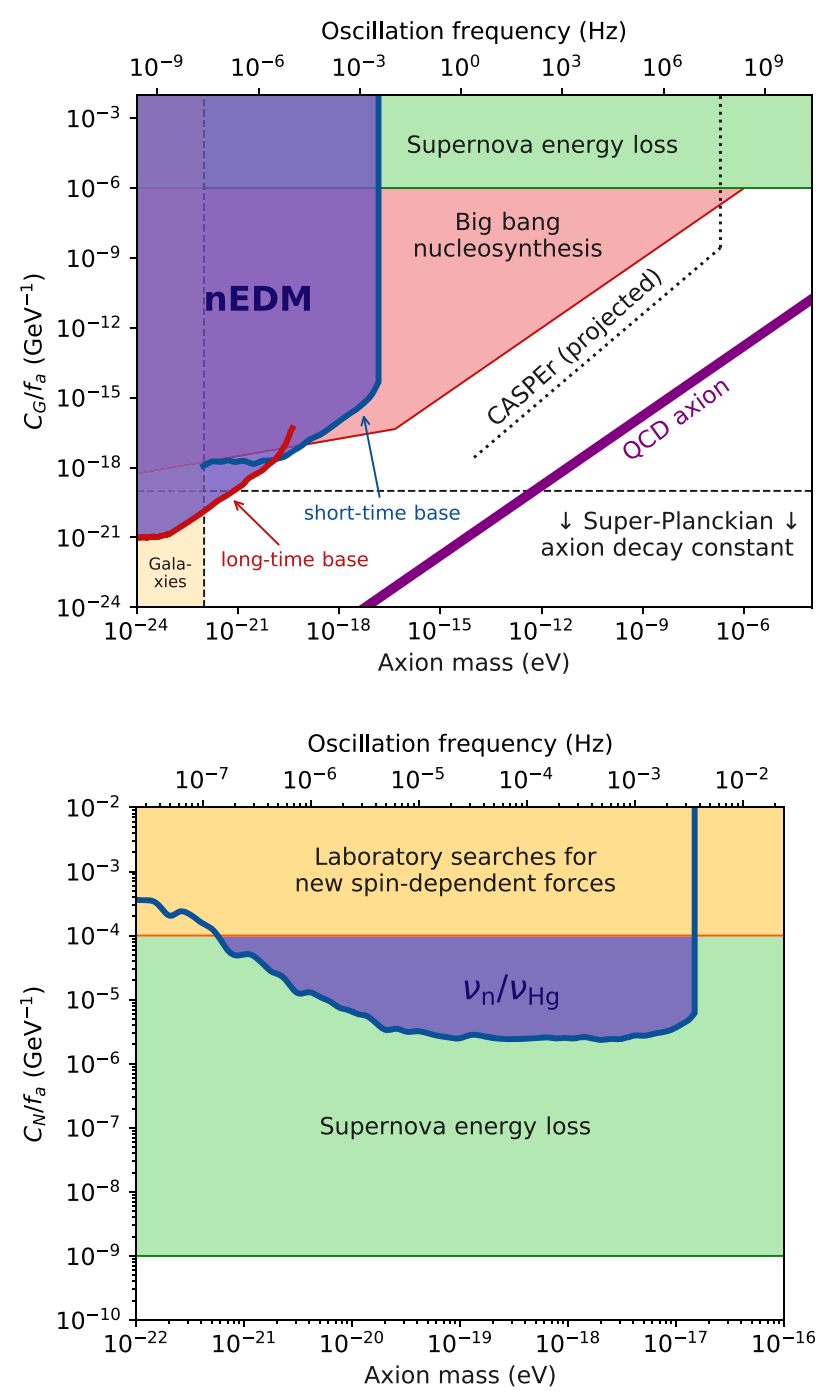

FIG. 4. Limits on the interactions of an axion with the gluons (top) and nucleons (bottom), as defined in Eq. (1), assuming that axions saturate the local cold DM content. The regions above the thick blue and red lines correspond to the regions of parameters excluded by the present work at the $95 \%$ confidence level (C.L.). The colored regions represent constraints from big bang nucleosynthesis (red, 95\% C.L.) [36-38], supernova energy-loss bounds (green, order of magnitude) $[35,39,40]$, consistency with observations of galaxies (orange) [15,25-27], and laboratory searches for new spin-dependent forces (yellow, 95\% C.L.) [41]. The $\mathrm{nEDM}, \nu_{n} / \nu_{\mathrm{Hg}}$, and big bang nucleosynthesis constraints scale as $\propto \sqrt{\rho_{a}}$, while the constraints from supernovae and laboratory searches for new spin-dependent forces are independent of $\rho_{a}$. The constraints from galaxies are relaxed if axions constitute a subdominant fraction of DM. We also show the projected reach of the proposed CASPEr experiment (dotted black line) [86], and the parameter space for the canonical QCD axion (purple band).

A nonstatistical excess in a periodogram of $R$ may be caused not only by a coherent oscillating signal; for example, fluctuations of a higher-order term in the magnetic field, not compensated by either the mercury or cesium magnetometers, may cause broadband elevations in LSSA power. We define strict requirements for an excess to be considered as one induced by axion DM as follows. Firstly, a significant $(>3 \sigma)$ excess in amplitude has to be observed in both sensitive data sets at the same frequency, but not in the control set. Secondly, the signals must be in antiphase in the parallel and antiparallel data sets. Lastly, we require high coherence (a narrow peak) equal to the spectral resolution of the data set. None of the significant excesses pass our discovery criteria.

We deliver a limit on the oscillation amplitude similarly to the long-time-base analysis, with the exception that we require the product of the two sensitive sets' $\mathrm{CL}_{s}$ statistics to be 0.05 . The limit is shown as the blue curve in Fig. 2 . With the short-time-base analysis, we are most sensitive to periods shorter than the time span of a sequence (2-3 days), and lose sensitivity to periods shorter than the cycle repetition rate $(\approx 5 \mathrm{~min})$. The PSI data set has a higher accumulated sensitivity than the ILL data set, so the limit baseline in the sensitive region is slightly better in the case of the PSI data set.

Following Eq. (2), we can interpret the limit on the oscillating neutron EDM as limits on the axion-gluon coupling in Eq. (1). We present these limits in Fig. 4, assuming that axions saturate the local cold DM energy density $\rho_{\mathrm{DM}}^{\text {local }} \approx 0.4 \mathrm{GeV} / \mathrm{cm}^{3}$ [55]. Our peak sensitivity is $f_{a} / C_{G} \approx 1 \times 10^{21} \mathrm{GeV}$ for $m_{a} \lesssim 10^{-23} \mathrm{eV}$, which probes super-Planckian axion decay constants $\left(f_{a}>M_{\text {Planck }} \approx\right.$ $10^{19} \mathrm{GeV}$ ), that is, interactions that are intrinsically feebler than gravity.

\section{AXION-WIND EFFECT}

We also perform a search for the axion-wind effect, Eq. (4), by partitioning the entire PSI data set into two sets with opposite magnetic-field orientations (irrespective of the electric field) and then analyzing the ratio $R=$ $\nu_{n} / \nu_{\mathrm{Hg}}$ similarly to our oscillating EDM analysis above. The axion-wind effect would manifest itself through time-dependent shifts in $\nu_{n}$ and $\nu_{\mathrm{Hg}}$ (and hence $R$ ) at three angular frequencies: $\omega_{1}=m_{a}, \omega_{2}=m_{a}+\Omega_{\text {sid }}$, and $\omega_{3}=\left|m_{a}-\Omega_{\text {sid }}\right|$, with the majority of power concentrated in the $\omega_{1}$ mode. Also, the axion-wind signal would have an opposite phase in the two subsets. We find two overlapping $3 \sigma$ excesses in the two subsets (at $3.42969 \mu \mathrm{Hz}$ and $3.32568 \mathrm{mHz}$ ), neither of which have a phase relation consistent with an axion-wind signal. Following Eq. (4), we derive limits on the axion-nucleon coupling in Eq. (1). We present these limits in Fig. 4, assuming that axions saturate the local cold DM energy density. Our peak sensitivity is $f_{a} / C_{N} \approx 4 \times 10^{5} \mathrm{GeV}$ for $10^{-19} \lesssim m_{a} \lesssim 10^{-17} \mathrm{eV}$.

\section{CONCLUSIONS}

In summary, we perform a search for a time-oscillating neutron EDM in order to probe the interaction of axionlike dark matter with gluons. We also perform a search for an axion-wind spin-precession effect in order to probe the 
interaction of axionlike dark matter with nucleons. So far, no significant oscillations have been detected, allowing us to place limits on the strengths of such interactions. Our limits improve upon existing astrophysical limits on the axion-gluon coupling by up to 3 orders of magnitude and also improve upon existing laboratory limits on the axion-nucleon coupling by up to a factor of 40 . Furthermore, we constrain a region of axion masses that is complementary to proposed "on-resonance" experiments in ferroelectrics [86]. Future EDM measurements will allow us to probe even feebler oscillations and for longer periods of oscillation that correspond to smaller axion masses.

\section{ACKNOWLEDGMENTS}

We are grateful to Maxim Pospelov for helpful discussions. The experimental data have been taken in part at the ILL Grenoble and at PSI Villigen. We acknowledge the excellent support by the technical groups of both institutions and by various services of the collaborating universities and research laboratories. Dedicated technical support by T. Brenner, M. Meier, and F. Burri is gratefully acknowledged. We remember with gratitude the pioneering contributions of Professor K. Smith and Professor J.M. Pendlebury, without whom these experiments could never have taken place. This work was funded in part by the U.K. Science and Technology Facilities Council (STFC) through Grants No. ST/N000307/1 and No. ST/M503836/1, as well as by the School of Mathematical and Physical Sciences at the University of Sussex. The original apparatus at ILL was funded by grants from the U.K.'s PPARC (now STFC), and we would like to thank the generations of engineers, students, and Research Fellows who contributed to its development. We gratefully acknowledge support of the Swiss National Science foundation under Grants No. 200020_172639, No. 20020_162574, No. 200020_163413, No. 200020_157079, and No. PP00P2_163663. This work has been supported in part by The National Science Centre, Poland, under Grant No. UMO2015/18/M/ST2/00056. This work has been supported by the Research Foundation-Flanders (FWO). The LPC Caen and the LPSC acknowledge the support of the French Agence Nationale de la Recherche under Reference No. ANR-09-BLAN-0046. M. F. was supported partly by the STFC Grant No. ST/L000326/1 and also by the European Research Council under the European Union's Horizon 2020 programme (ERC project 648680 DARKHORIZONS). V. V.F. was supported by the Gutenberg Research College Fellowship and by the Australian Research Council. D. J.E. M. was supported by a Royal Astronomical Society postdoctoral fellowship hosted at King's College London. P. M. was supported by the State Secretariat for Education, Research and Innovation (SERI) - Federal Commission for Scholarships for Foreign Students (FCS) Grant No. 2015.0594. Y. V. S. was supported by the Humboldt Research Fellowship and in part by the Australian Research Council. E. W. was supported by a Ph.D. Fellowship of the Research Foundation Flanders (FWO).

[1] P. A. R. Ade et al. (Planck Collaboration), Planck 2015 Results-XIII. Cosmological Parameters, Astron. Astrophys 594, A13 (2016).

[2] R. D. Peccei and H. R. Quinn, CP Conservation in the Presence of Pseudoparticles, Phys. Rev. Lett. 38, 1440 (1977).

[3] R. D. Peccei and H. R. Quinn, Constraints Imposed by CP Conservation in the Presence of Pseudoparticles, Phys. Rev. D 16, 1791 (1977).

[4] S. Weinberg, A New Light Boson?, Phys. Rev. Lett. 40, 223 (1978).

[5] F. Wilczek, Problem of Strong $P$ and T Invariance in the Presence of Instantons, Phys. Rev. Lett. 40, 279 (1978).

[6] J. E. Kim, Weak-Interaction Singlet and Strong CP Invariance, Phys. Rev. Lett. 43, 103 (1979).

[7] M. A. Shifman, A. I. Vainshtein, and V. I. Zakharov, Can Confinement Ensure Natural CP Invariance of Strong Interactions?, Nucl. Phys. B166, 493 (1980).

[8] A. R. Zhitnitsky, The Weinberg Model of the CP Violation and T Odd Correlations in Weak Decays, Yad. Fiz. 31, 1024 (1980) [Sov. J. Nucl. Phys. 31, 529 (1980)].

[9] M. Dine, W. Fischler, and M. Srednicki, A Simple Solution to the Strong CP Problem with a Harmless Axion, Phys. Lett. B 104, 199 (1981).

[10] E. Witten, Some Properties of O(32) Superstrings, Phys. Lett. B 149, 351 (1984).

[11] J. P. Conlon, The QCD Axion and Moduli Stabilisation, J. High Energy Phys. 05 (2006) 078.

[12] P. Svrcek and E. Witten, Axions in String Theory, J. High Energy Phys. 06 (2006) 051.

[13] A. Arvanitaki, S. Dimopoulos, S. Dubovsky, N. Kaloper, and J. March-Russell, String Axiverse, Phys. Rev. D 81, 123530 (2010).

[14] P. Arias, D. Cadamuro, M. Goodsell, J. Jaeckel, J. Redondo, and A. Ringwald, Wispy Cold Dark Matter, J. Cosmol. Astropart. Phys. 06 (2012) 013.

[15] D. J. E. Marsh, Axion Cosmology, Phys. Rep. 643, 1 (2016).

[16] J. Preskill, M. B. Wise, and F. Wilczek, Cosmology of the Invisible Axion, Phys. Lett. B 120, 127 (1983).

[17] L. F. Abbott and P. Sikivie, A Cosmological Bound on the Invisible Axion, Phys. Lett. B 120, 133 (1983).

[18] M. Dine and W. Fischler, The Not-So-Harmless Axion, Phys. Lett. B 120, 137 (1983).

[19] Although nonthermal production mechanisms typically impart negligible kinetic energy to the produced axions, the gravitational interactions between axions and ordinary matter during galactic structure formation subsequently virialize galactic axions $\left(v_{\text {vir }}^{\text {local }} \sim 300 \mathrm{~km} / \mathrm{s}\right.$ ), giving an oscillating galactic axion field the finite coherence time: $\tau_{\mathrm{coh}} \sim 2 \pi / m_{a} v_{\mathrm{vir}}^{2} \sim 2 \pi \times 10^{6} / m_{a}$, i.e., $\Delta \omega / \omega \sim 10^{-6}$.

[20] M. Yu. Khlopov, B. A. Malomed, and Ya. B. Zeldovich, Gravitational Instability of Scalar Fields and Formation of 
Primordial Black Holes, Mon. Not. R. Astron. Soc. 215, 575 (1985).

[21] W. Hu, R. Barkana, and A. Gruzinov, Fuzzy Cold Dark Matter: The Wave Properties of Ultralight Particles, Phys. Rev. Lett. 85, 1158 (2000).

[22] D. J. E. Marsh and J. Silk, A Model for Halo Formation with Axion Mixed Dark Matter, Mon. Not. R. Astron. Soc. 437, 2652 (2014).

[23] H.-Y. Schive, T. Chiueh, and T. Broadhurst, Cosmic Structure as the Quantum Interference of a Coherent Dark Wave, Nat. Phys. 10, 496 (2014).

[24] L. Hui, J. P. Ostriker, S. Tremaine, and E. Witten, Ultralight Scalars as Cosmological Dark Matter, Phys. Rev. D 95, 043541 (2017).

[25] B. Bozek, D. J. E. Marsh, J. Silk, and R. F. G. Wyse, Galaxy UV-Luminosity Function and Reionization Constraints on Axion Dark Matter, Mon. Not. R. Astron. Soc. 450, 209 (2015).

[26] H.-Y. Schive, T. Chiueh, T. Broadhurst, and K.-W. Huang, Contrasting Galaxy Formation from Quantum Wave Dark Matter, $\psi \mathrm{DM}$, with $\Lambda \mathrm{CDM}$, Using Planck and Hubble Data, Astrophys. J. 818, 89 (2016).

[27] P. S. Corasaniti, S. Agarwal, D. J. E. Marsh, and S. Das, Constraints on Dark Matter Scenarios from Measurements of the Galaxy Luminosity Function at High Redshifts, Phys. Rev. D 95, 083512 (2017).

[28] R. Hlozek, D. Grin, D. J. E. Marsh, and P. G. Ferreira, A Search for Ultralight Axions Using Precision Cosmological Data, Phys. Rev. D 91, 103512 (2015).

[29] P. W. Graham, I. G. Irastorza, S. K. Lamoreaux, A. Lindner, and K. A. van Bibber, Experimental Searches for the Axion and Axion-like Particles, Annu. Rev. Nucl. Part. Sci. 65, 485 (2015).

[30] P. W. Graham and S. Rajendran, Axion Dark Matter Detection with Cold Molecules, Phys. Rev. D 84, 055013 (2011).

[31] Y. V. Stadnik and V. V. Flambaum, Axion-Induced Effects in Atoms, Molecules, and Nuclei: Parity Nonconservation, Anapole Moments, Electric Dipole Moments, and SpinGravity and Spin-Axion Momentum Couplings, Phys. Rev. D 89, 043522 (2014).

[32] B. M. Roberts, Y. V. Stadnik, V. A. Dzuba, V. V. Flambaum, $\mathrm{N}$. Leefer, and D. Budker, Limiting p-Odd Interactions of Cosmic Fields with Electrons, Protons, and Neutrons, Phys. Rev. Lett. 113, 081601 (2014).

[33] B. M. Roberts, Y. V. Stadnik, V. A. Dzuba, V. V. Flambaum, N. Leefer, and D. Budker, Parity-Violating Interactions of Cosmic Fields with Atoms, Molecules, and Nuclei: Concepts and Calculations for Laboratory Searches and Extracting Limits, Phys. Rev. D 90, 096005 (2014).

[34] V. V. Flambaum, in Proceedings of the 9th Patras Workshop on Axions, WIMPs and WISPs, Mainz, Germany, 2013, http://axion-wimp2013.desy.de/e201031/index_eng.html.

[35] P. W. Graham and S. Rajendran, New Observables for Direct Detection of Axion Dark Matter, Phys. Rev. D 88, 035023 (2013).

[36] K. Blum, R. T. D'Agnolo, M. Lisanti, and B. R. Safdi, Constraining Axion Dark Matter with Big Bang Nucleosynthesis, Phys. Lett. B 737, 30 (2014).

[37] Y. V. Stadnik, Manifestations of Dark Matter and Variations of the Fundamental Constants of Nature in Atoms and
Astrophysical Phenomena, (Springer, Cham, Switzerland, 2017).

[38] Y. V. Stadnik and V. V. Flambaum, Can Dark Matter Induce Cosmological Evolution of the Fundamental Constants of Nature?, Phys. Rev. Lett. 115, 201301 (2015).

[39] G. G. Raffelt, Astrophysical Methods to Constrain Axions and Other Novel Particle Phenomena, Phys. Rep. 198, 1 (1990).

[40] G. G. Raffelt, in Axions: Theory, Cosmology, and Experimental Searches, edited by M. Kuster, G. Raffelt, and B. Beltrán (Springer, Berlin, 2008), pp. 51-71.

[41] G. Vasilakis, J. M. Brown, T. W. Kornack, and M. V. Romalis, Limits on New Long Range Nuclear SpinDependent Forces Set with a $K-{ }^{3} \mathrm{He}$ Comagnetometer, Phys. Rev. Lett. 103, 261801 (2009).

[42] Interaction [Eq. (1)] also nonperturbatively induces a mass $m_{a} \approx 6 C_{G} \mu \mathrm{eV} \times\left(10^{12} \mathrm{GeV} / f_{a}\right)$. In order for an axion to have a smaller mass, a fine-tuning mechanism (e.g., a tachyonic mass contribution [36]) is required to cancel the nonperturbative mass contribution.

[43] R. J. Crewther, P. Di Vecchia, G. Veneziano, and E. Witten, Chiral Estimate of the Electric Dipole Moment of the Neutron in Quantum Chromodynamics, Phys. Lett. B 88, 123 (1979); Chiral Estimate of the Electric Dipole Moment of the Neutron in Quantum Chromodynamics, Phys. Lett. B 91, 487(E) (1980).

[44] M. Pospelov and A. Ritz, Theta-Induced Electric Dipole Moment of the Neutron via QCD Sum Rules, Phys. Rev. Lett. 83, 2526 (1999).

[45] O. P Sushkov, V. V. Flambaum, and I. B. Khriplovich, Possibility of Investigating P-and T-Odd Nuclear Forces in Atomic and Molecular Experiments, Zh. Eksp. Teor. Fiz. 87, 1521 (1984) [Sov. Phys. JETP 60, 873 (1984)].

[46] V. V. Flambaum, I. B. Khriplovich, and O. P. Sushkov, Limit on the Constant of T-Nonconserving Nucleon-Nucleon Interaction, Phys. Lett. B 162, 213 (1985).

[47] V. V. Flambaum, I. B. Khriplovich, and O. P. Sushkov, On the $P$ - and T-Nonconserving Nuclear Moments, Nucl. Phys. A449, 750 (1986).

[48] V. A. Dzuba, V. V. Flambaum, J. S. M. Ginges, and M. G. Kozlov, Electric Dipole Moments of $\mathrm{Hg}, \mathrm{Xe}, \mathrm{Rn}, \mathrm{Ra}, \mathrm{Pu}$, and TIF Induced by the Nuclear Schiff Moment and Limits on Time-Reversal Violating Interactions, Phys. Rev. A 66, 012111 (2002).

[49] V. F. Dmitriev and R. A. Sen'kov, Schiff Moment of the Mercury Nucleus and the Proton Dipole Moment, Phys. Rev. Lett. 91, 212303 (2003).

[50] V. F. Dmitriev and R. A. Sen'kov, P-and T-Violating Schiff Moment of the Mercury Nucleus, Phys. At. Nucl. 66, 1940 (2003).

[51] V. F. Dmitriev, R. A. Sen'kov, and N. Auerbach, Effects of Core Polarization on the Nuclear Schiff Moment, Phys. Rev. C 71, 035501 (2005).

[52] J. H. de Jesus and J. Engel, Time-Reversal-Violating Schiff Moment of ${ }^{199} \mathrm{Hg}$, Phys. Rev. C 72, 045503 (2005).

[53] S. Ban, J. Dobaczewski, J. Engel, and A. Shukla, Fully Self-Consistent Calculations of Nuclear Schiff Moments, Phys. Rev. C 82, 015501 (2010).

[54] L.I. Schiff, Measurability of Nuclear Electric Dipole Moments, Phys. Rev. 132, 2194 (1963). 
[55] R. Catena and P. Ullio, A Novel Determination of the Local Dark Matter Density, J. Cosmol. Astropart. Phys. 08 (2010) 004.

[56] V. A. Kostelecký and C. D. Lane, Constraints on Lorentz Violation from Clock-Comparison Experiments, Phys. Rev. D 60, 116010 (1999).

[57] NASA LAMBDA-Tools, http://lambda.gsfc.nasa.gov/ toolbox/tb_coordconv.cfm, accessed July 25, 2017.

[58] Y. V. Stadnik and V. V. Flambaum, Nuclear Spin-Dependent Interactions: Searches for WIMP, Axion and Topological Defect Dark Matter, and Tests of Fundamental Symmetries, Eur. Phys. J. C 75, 110 (2015).

[59] I. Altarev et al., Test of Lorentz Invariance with Spin Precession of Ultracold Neutrons, Phys. Rev. Lett. 103, 081602 (2009).

[60] I. Altarev et al., New Constraints on Lorentz Invariance Violation from the Neutron Electric Dipole Moment, Europhys. Lett. 92, 51001 (2010).

[61] S. Afach et al., Constraining Interactions Mediated by Axion-like Particles with Ultracold Neutrons, Phys. Lett. B 745, 58 (2015).

[62] C. A. Baker et al., Apparatus for Measurement of the Electric Dipole Moment of the Neutron Using a Cohabiting Atomic-Mercury Magnetometer, Nucl. Instrum. Methods Phys. Res., Sect. A 736, 184 (2014).

[63] C. A. Baker et al., The Search for the Neutron Electric Dipole Moment at the Paul Scherrer Institute, Speical Issue on 2nd International Workshop on the Physics of Fundamental Symmetries and Interactions-PSI2010, Phys. Procedia 17, 159 (2011).

[64] C. A. Baker et al., Improved Experimental Limit on the Electric Dipole Moment of the Neutron, Phys. Rev. Lett. 97, 131801 (2006); Baker et al. Reply, Phys. Rev. Lett. 98, 149102 (2007).

[65] J. M. Pendlebury et al., Revised Experimental Upper Limit on the Electric Dipole Moment of the Neutron, Phys. Rev. D 92, 092003 (2015).

[66] N. F. Ramsey, A Molecular Beam Resonance Method with Separated Oscillating Fields, Phys. Rev. 78, 695 (1950).

[67] R. Golub and J. M. Pendlebury, Ultra-Cold Neutrons, Rep. Prog. Phys. 42, 439 (1979).

[68] R. Golub and S. K. Lamoreaux, Neutron Electric-Dipole Moment, Ultracold Neutrons and Polarized ${ }^{3} \mathrm{He}$, Phys. Rep. 237, 1 (1994).

[69] A. Steyerl, H. Nagel, F.-X. Schreiber, K.-A. Steinhauser, R. Gähler, W. Gläser, P. Ageron, J. M. Astruc, W. Drexel, G. Gervais, and W. Mampe, A New Source of Cold and Ultracold Neutrons, Phys. Lett. A 116, 347 (1986).

[70] S. Afach et al., A Measurement of the Neutron to ${ }^{199} \mathrm{Hg}$ Magnetic Moment Ratio, Phys. Lett. B 739, 128 (2014).

[71] J. M. Pendlebury, W. Heil, Y. Sobolev, P. G. Harris, J. D. Richardson, R. J. Baskin, D. D. Doyle, P. Geltenbort,
K. Green, M. G. D. van der Grinten, P. S. Iaydjiev, S. N. Ivanov, D. J. R. May, and K. F. Smith, Geometric-PhaseInduced False Electric Dipole Moment Signals for Particles in Traps, Phys. Rev. A 70, 032102 (2004).

[72] J. D. Scargle, Studies in Astronomical Time Series Analysis. II-Statistical Aspects of Spectral Analysis of Unevenly Spaced Data, Astrophys. J. 263, 835 (1982).

[73] A. Cumming, Detectability of Extrasolar Planets in Radial Velocity Surveys, Mon. Not. R. Astron. Soc. 354, 1165 (2004).

[74] S. Algeri, D. A. van Dyk, J. Conrad, and B. Anderson, On Methods for Correcting for the Look-Elsewhere Effect in Searches for New Physics, J. Instrum. 11, P12010 (2016).

[75] C. Patrignani et al. (Particle Data Group), Review of Particle Physics, Chin. Phys. C 40, 100001 (2016).

[76] A. Anghel et al., The PSI Ultra-Cold Neutron Source, Nucl. Instrum. Methods Phys. Res., Sect. A 611, 272 (2009).

[77] B. Lauss, A New Facility for Fundamental Particle Physics: The High-Intensity Ultracold Neutron Source at the Paul Scherrer Institute, AIP Conf. Proc. 1441, 576 (2012).

[78] B. Lauss, Startup of the High-Intensity Ultracold Neutron Source at the Paul Scherrer Institute, Hyperfine Interact. 211, 21 (2012).

[79] B. Lauss, Ultracold Neutron Production at the Second Spallation Target of the Paul Scherrer Institute, Phys. Procedia 51, 98 (2014).

[80] S. Afach et al., A Device for Simultaneous Spin Analysis of Ultracold Neutrons, Eur. Phys. J. A 51, 143 (2015).

[81] G. Ban et al., Ultracold Neutron Detection with ${ }^{6}$ Li-Doped Glass Scintillators, Eur. Phys. J. A 52, 326 (2016).

[82] P. Knowles, G. Bison, N. Castagna, A. Hofer, A. Mtchedlishvili, A. Pazgalev, and A. Weis, Laser-Driven Cs Magnetometer Arrays for Magnetic Field Measurement and Control, Special Issue on Particle Physics with Slow Neutrons, Nucl. Instrum. Methods Phys. Res., Sect. A 611, 306 (2009).

[83] H.-C. Koch, G. Bison, Z. D. Grujić, W. Heil, M. Kasprzak, P. Knowles, A. Kraft, A. Pazgalev, A. Schnabel, J. Voigt, and A. Weis, Investigation of the Intrinsic Sensitivity of a ${ }^{3} \mathrm{He} / \mathrm{Cs}$ Magnetometer, Eur. Phys. J. D 69, 262 (2015).

[84] S. Afach et al., Observation of Gravitationally Induced Vertical Striation of Polarized Ultracold Neutrons by Spin-Echo Spectroscopy, Phys. Rev. Lett. 115, 162502 (2015).

[85] E. Wursten (to be published).

[86] D. Budker, P. W. Graham, M. Ledbetter, S. Rajendran, and A. O. Sushkov, Proposal for a Cosmic Axion Spin Precession Experiment (CASPEr), Phys. Rev. X 4, 021030 (2014). 